\title{
Dense Sphere Packings from Optimized Correlation Functions
}

\author{
Adam B. Hopkins and Frank H. Stillinger \\ Department of Chemistry; Princeton University, Princeton, New Jersey 08544 \\ Salvatore Torquato \\ Department of Chemistry, Princeton Institute for the Science and Technology of Materials, \\ Program in Applied and Computational Mathematics, \\ Princeton Center for Theoretical Science; Princeton University, \\ Princeton, New Jersey 08544; School of Natural Sciences, \\ Institute for Advanced Study; Princeton, New Jersey 08544
}

\begin{abstract}
Elementary smooth functions (beyond contact) are employed to construct pair correlation functions that mimic jammed disordered sphere packings. Using the $g_{2}$-invariant optimization method of Torquato and Stillinger [J. Phys. Chem. B 106, 8354, 2002], parameters in these functions are optimized under necessary realizability conditions to maximize the packing fraction $\phi$ and average number of contacts per sphere $Z$. A pair correlation function that incorporates the salient features of a disordered packing and that is smooth beyond contact is shown to permit a $\phi$ of 0.6850 : this value represents a $45 \%$ reduction in the difference between the maximum for congruent hard spheres in three dimensions, $\pi / \sqrt{18} \approx 0.7405$, and 0.64 , the approximate fraction associated with maximally random jammed (MRJ) packings in three dimensions. We show that, surprisingly, the continued addition of elementary functions consisting of smooth sinusoids decaying as $r^{-4}$ permits packing fractions approaching $\pi / \sqrt{18}$. A translational order metric is used to discriminate between degrees of order in the packings presented. We find that to achieve higher packing fractions, the degree of order must increase, which is consistent with the results of a previous study [Torquato et al., Phys. Rev. Lett. 84, 2064, 2000].

PACS numbers:
\end{abstract}




\section{INTRODUCTION AND BACKGROUND}

Packing problems address the various arrangements of a set (finite or infinite) of nonoverlapping objects in a space of given dimension [1, 2, 3]. Often, one seeks to arrange the objects in such a way as to optimize a certain statistical or bulk property, e.g. the number density $\rho$ of objects (or equivalently the packing fraction $\phi$, the fraction of space covered by the objects' interiors). This paper is concerned with optimizing the packing fraction $\phi$ and average number of contacts per sphere (average kissing number) $Z$ for packings of congruent spheres in three-dimensional Euclidean space while maintaining an assumed functional form for the pair correlation function (defined below) of a "random" packing.

Random packings of three-dimensional hard spheres have been studied by scientists to better understand everything from heterogeneous materials to liquids to granular media (like sand) to living cells [3, 4, 5, 6, 17]. In parallel to the concept of a maximum packing fraction for periodic (crystalline) packings, it had been assumed that a (different) maximum packing fraction could be defined for random packings, referred to as "random close packing" (RCP) [8, 9]. However, while there is a proved maximum packing fraction for hard-sphere periodic packings achieved via an FCC lattice or one of its stacking variants [2], the RCP state has been shown to be ill-defined [10, 11].

Experimental packings of oiled steel ball bearings originally led to the idea that mechanically stable random packings of identical spheres could not exhibit packing fractions exceeding 0.64 or declining past 0.60 [9, 12]. Mathematically constructed models [13] and early computer simulations [14] seemed to support these conclusions, though later work demonstrated that the limiting packing fractions obtained were highly dependent on the packing methods [3, 10]. These methods included, for example, lightly vibrating a container filled with spheres in either horizontal or vertical motions, rolling spheres one by one into a container [15], and simulating the compression of a hard-sphere gas [16, 17].

Changing the method of packing via molecular dynamics simulations showed that densities past 0.64 are realizable [10, 18, 19]. However, it has yet to be demonstrated from a theoretical basis, i.e., without resort to experiment or computer simulation, that indeed there is no maximum density limit for "random" packings apart from the proved limit for periodic packings. This paper provides such a theoretical basis by extending previous optimization studies of $g_{2}$-invariant processes (defined below) [20, 21] to a broader class of 
disordered packings.

Following previous work by two of us, a statistically homogeneous and isotropic packing is defined to be disordered if its pair correlation function $g_{2}(r)$ in $d$-dimensional Euclidean space $\mathbb{R}^{d}$ decays to unity faster than $r^{-d-\epsilon}$ for some $\epsilon>0$ [21]. Each packing corresponds to a unique $g_{2}(r)$, a function proportional to the probability density of finding a separation $r$ between any two sphere centers and normalized such that it takes the value of unity when no spatial correlations are present. The precise definition of "disordered" via the pair correlation function takes the place of the imprecise term "random" hereafter.

The essential ideas behind our approach were actually laid out in our earlier work [20, 21]. In Ref. [20], the main objective was to study disordered packings in which short-range order was controlled using so-called $g_{2}$-invariant processes. A $g_{2}$-invariant process is one in which a given pair correlation function $g_{2}(r)$ remains invariant for all $r$ as packing fraction varies over the range of densities

$$
0 \leq \phi \leq \phi_{*}
$$

The terminal packing fraction $\phi_{*}$ is the maximum achievable for the $g_{2}$-invariant process subject to satisfaction of the nonnegativity of $g_{2}(r)$ and the structure factor $S(k)$, i.e.,

$$
\begin{aligned}
& g_{2}(r) \geq 0 \quad \forall r \geq 0 \\
& S(k) \geq 0 \quad \forall k \geq 0 .
\end{aligned}
$$

For a statistically homogeneous and isotropic packing at number density $\rho, S(k)$ is related to the Fourier transform of the total correlation function $h(r) \equiv g_{2}(r)-1$, by

$$
S(k)=1+\rho \tilde{h}(k)
$$

where $\tilde{h}(k)$ represents the Fourier transform of $h(r)$. In three dimensions, this can be written as

$$
S(k)=1+4 \pi \rho \int_{0}^{\infty} \frac{r \sin k r}{k} h(r) d r .
$$

The optimization procedure described above was formulated for a hard-sphere packing, which requires the additional condition on $g_{2}$ that respects the nonoverlap constraint, i.e.,

$$
g_{2}(r)=0 \quad \text { for } \quad 0 \leq r<D
$$

When there exist sphere packings with a $g_{2}$ satisfying conditions (2), (3), and (6) for $\phi$ in the interval $\left[0, \phi_{*}\right]$, then a lower bound on the maximal packing fraction is given by 
$\phi_{\max } \geq \phi_{*} . \quad$ It is noteworthy that this optimization problem for sphere packings is an infinite-dimensional linear program, which is the dual of the primal linear program devised by Cohn and Elkies [22] to obtain upper bounds on the maximal packing fraction [21]. We will comment further on this connection in the conclusions. Finally, we note here that the results of the optimization of the pair correlation function given in Ref. [20] has found application in describing small-scale convective structural features of the solar surface [23].

The nonnegativity conditions (2) and (3) are necessary, but generally not sufficient, for a pair correlation function at a given density to be realizable by a point process [24]. A third condition, obtained by Yamada [25] and not included in the optimization procedure described above, constrains $\sigma^{2}(A) \equiv\left\langle(N(A)-\langle N(A)\rangle)^{2}\right\rangle$, which is the variance in the number of points $N(A)$ contained within a window $A \in \mathbb{R}^{d}$;

$$
\sigma^{2}(A) \geq \theta(A)[1-\theta(A)]
$$

where $\theta(A)$ is the fractional part of the expected number of points contained within the window. The number variance associated with a spherical window of radius $R$ for a statistically homogeneous point process in $d$ dimensions can be written as follows [26]:

$$
\sigma^{2}(R)=\rho v_{1}(R)\left[1+\rho \int_{\mathbb{R}^{d}} h(\mathbf{r}) \alpha_{2}^{i n t}(r ; R) d \mathbf{r}\right] \geq \theta(R)[1-\theta(R)]
$$

where $v_{1}(R)$ is the volume of the window and $\alpha_{2}^{i n t}(r ; R)$ the intersection volume of two windows of radius $R$ (whose centers are separated by $\mathbf{r}$ ) divided by $v_{1}(R)$. This additional condition (8) on the pair correlation function is always satisfied for statistically homogeneous and isotropic packings with sufficiently large windows in dimensions greater than 1 [21]. In all cases that have been studied, this condition is satisfied for all $R$ if the first two conditions are satisfied [21, 27].

While conditions (2), (3), and (7) are necessary for the realizability of point processes, along with incorporation in $g_{2}(r)$ of the core exclusion feature, they appear to be rather strong conditions for realizability of sphere packings, especially as the space dimension increases [21]. For example, a method to construct disordered packing configurations that realize test $g_{2}$ 's meeting the conditions and incorporating the features of core exclusion and contact pairs [Eqs. (9) and (11)] has been successful [28, 29] in two and three dimensions. No example in three dimensions or greater of an unrealizable $g_{2}$ satisfying the conditions and incorporating the core exclusion feature is currently known. 
In Ref. [20], a five-parameter test family of $g_{2}$ 's incorporating features of core exclusion, contact pairs, and damped oscillatory short-range order beyond contact [Eqs. (9), (11), and $(12)]$ had been considered. The problem of finding the terminal packing fraction $\phi_{*}$ was posed as an optimization problem: maximize $\phi$ over the set of parameters subject to the first two realizability conditions (the third condition due to Yamada was not relevant). In this work, we consider a broader family of smooth $g_{2}$ test functions corresponding to disordered packings [30] and satisfying all three aforementioned conditions.

To demonstrate the absence of a theoretical upper limit on disordered packings, we show that terms decaying as $r^{-4}$, representative of a feature prominent in the pair correlation functions of maximally random jammed (MRJ) packings [31], allow for increased packing fraction for pair correlation functions satisfying the three conditions and incorporating the aforementioned features. A simple 11-parameter form consisting of the initial five-parameter form plus two sinusoids decaying as $r^{-4}$ permits a packing fraction of 0.6850 . Using a translational order metric, we show that the pair correlation function with the highest packing fraction also exhibits the highest degree of order, which is consistent with the conclusions of a previous work [10]. Additionally we show the surprising result that the continued addition of terms decaying as $r^{-4}$ allows for packing fractions up to $\pi / \sqrt{18}$, indicating that, if the packings are realizable, the progression of disordered packings up to the maximum $\phi$ is a continuum, dependent only on the form and parameters of the functions employed. A qualitative description of a realizable disordered packing with smooth $g_{2}(r)$ and $\phi$ approaching $\pi / \sqrt{18}$ is provided in Section IV A.

\section{OPTIMIZATION OF $g_{2}$-INVARIANT PROCESSES}

We begin by revisiting the optimization problem first examined in Ref. [20]. We employ a more comprehensive search using simulated annealing to optimize the five parameters of the family of $g_{2}$ 's presented in Ref. [20] and find a higher terminal packing fraction $\phi_{*}=0.64268$. The three functions that comprise the five parameter family, $g_{I}(r), g_{I I}(r)$ and $g_{I I I}(r)$, capture the most salient properties of a disordered packing, including that the average number of spheres in contact is $Z$ and that no sphere centers may approach closer than a distance of one sphere diameter. 
A Heaviside step function represents the spheres' hard core exclusion,

$$
g_{I}=\Theta(r-1)
$$

where we set the diameter of the spheres to be unity. The Heaviside step function $\Theta(x)$ is defined piecewise as

$$
\Theta(x)= \begin{cases}0, & x<0 \\ 1, & x \geq 0 .\end{cases}
$$

A Dirac delta function represents pair contacts,

$$
g_{I I}=\frac{Z}{4 \pi \rho} \delta(r-1),
$$

with $Z$ the average number of contacts per sphere (average kissing number). An exponentially decaying sinusoid provides short-range oscillatory motion about unity:

$$
g_{I I I}=\frac{A_{1}}{r} \exp \left(-B_{1} r\right) \sin \left(C_{1} r+D_{1}\right) \Theta(r-1),
$$

with parameters $A_{1}, B_{1}, C_{1}, D_{1}$. The total pair correlation function $g_{2}(r)$ is then

$$
g_{2}(r)=g_{I}(r)+g_{I I}(r)+g_{I I I}(r) .
$$

Constraining $B_{1}>0, Z \geq 0$ ensures a physical configuration, while constraining $C_{1} \geq 0$,

$0 \leq D_{1}<\pi$ eliminates function duplicates without additionally constraining the range of the functional form.

\section{A. Maximizing packing fraction}

Packing fraction is maximized using the $g_{2}$-invariant method in 20,000 independent runs of over 10,000 iterations each. Initial parameters are confined to the following bounds, and selected randomly before each run with exponentially decreasing probability from zero:

$$
\begin{gathered}
-50<A_{1}<100 \\
0<B_{1}<10 \\
0 \leq C_{1}<50 \\
0 \leq D_{1}<\pi \\
0 \leq Z<13
\end{gathered}
$$


Parameters are allowed to range outside of bounds, but in no cases of the 20,000 did this occur.

Using $G_{I}(k), G_{I I}(k), G_{I I I}(k)$ to represent $1 / \rho$ times the second term on the right hand side of relation (5) with $h(r)=g_{I}(r)+g_{I I}(r)+g_{I I I}(r)-1$, the structure factor for the functions becomes

$$
S(k)=1+\frac{Z \sin (k)}{k}+\rho\left(G_{I}(k)+G_{I I I}(k)\right),
$$

with $G_{I I}(k)=Z \sin (k) / \rho k$. The exact analytical forms for $G_{I}, G_{I I}$, and $G_{I I I}$ are included in Appendix B.

The method to maximize $\phi$ relies upon a simple principal. If $S(K)=0$ at some point $K$ and $G_{I}(K)+G_{I I I}(K)<0$, while $S(k) \geq 0$ for all other points $k$, then $\phi$ is at a global maximum for the given five parameters, i.e., $\phi=\phi_{*}$. Hence to maximize $\phi_{*}, S(k)$ is analytically calculated from the pair correlation function in accordance with Eq. (14), and for each random step along one of the five parameters, if possible $\phi$ is chosen such that the structure factor is in accordance with this principal. If obeying the structure factor condition is not mathematically possible for the parameter set, if the pair correlation function $g_{2}(r)$ is not greater than or equal to zero for all $r$, or if the maximum $\phi$ for the set does not meet the standard temperature-dependent simulated annealing condition for accepting a move, the random step is rejected and a new step chosen.

Figures 1 and 2 present pair correlation functions and corresponding structure factors that yield terminal packing fractions $\phi_{*}=0.64268$ and $\phi_{*}=0.64050$, respectively. Parameters for high packing-fraction results were similar in period $C_{1}$, phase $D_{1}$, and average kissing number $Z$, but varied in amplitude $A_{1}$ and damping factor $B_{1}$. For example, the $g_{2}(r)$ with the highest two terminal packing fractions, $\phi_{*}=0.64268$ and $\phi_{*}=0.64050$, exhibited $A_{1}=3.0996$ and $A_{1}=6.1707$, with $B_{1}=0.58091$ and $B_{1}=1.2090$, respectively. Each $g_{2}(r)$ exhibits a minimum near $r=1.35$, with the minimum for the highest packing fraction equal to zero, and a maximum at $r=1$ of about 2.7, suggesting that these traits, along with a period $2 \pi / C_{1}$ of about 1.2 and a phase $D_{1}$ of about 0.50 are important in obtaining the maximum packing fraction for this functional form. Further analysis indicates that the period and existence of a deep minimum within $r \approx 1.5$ remain important in maximizing packing fraction when other elements are added to this functional form. 

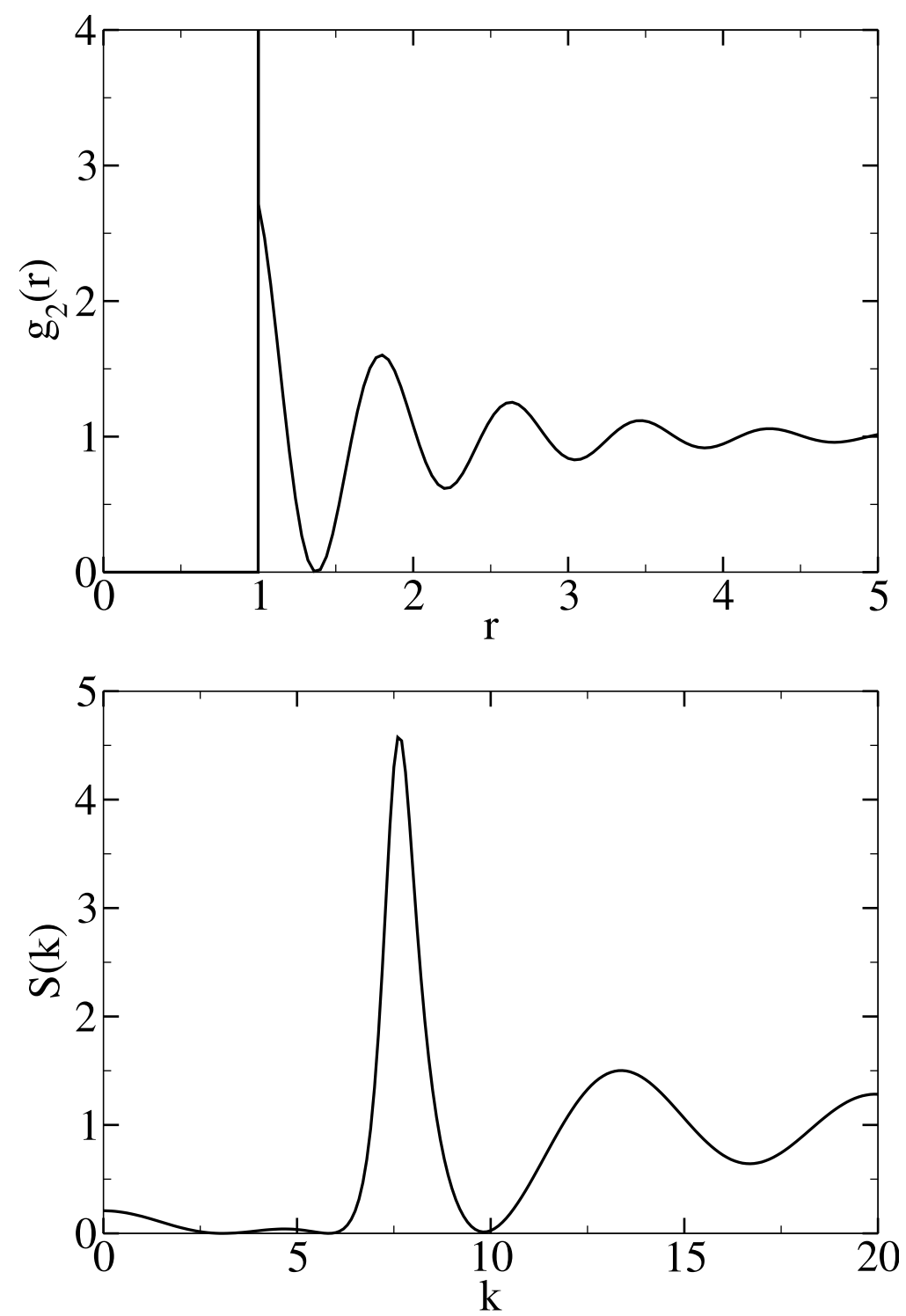

FIG. 1: Top: The pair correlation function $g_{2}(r)$ for $\phi_{*}=0.64268, A_{1}=3.0996, B_{1}=0.58091$, $C_{1}=7.54069, D_{1}=0.45970, Z=5.0633$. Bottom: The corresponding structure factor $S(k)$.

\section{B. Maximizing kissing number}

Maximum packing-fraction $g_{2}$ 's for this functional form do not correspond to $g_{2}$ 's that maximize average kissing number, as is the general case for sphere packings in many dimensions [27. Moreover, though the average kissing number for the highest possible packing fraction (FCC lattice) is 12 [32], the proved maximum possible, $Z$ may only obtain the value of 9.5401 for this form. As will be seen later, as additional elements and parameters are 

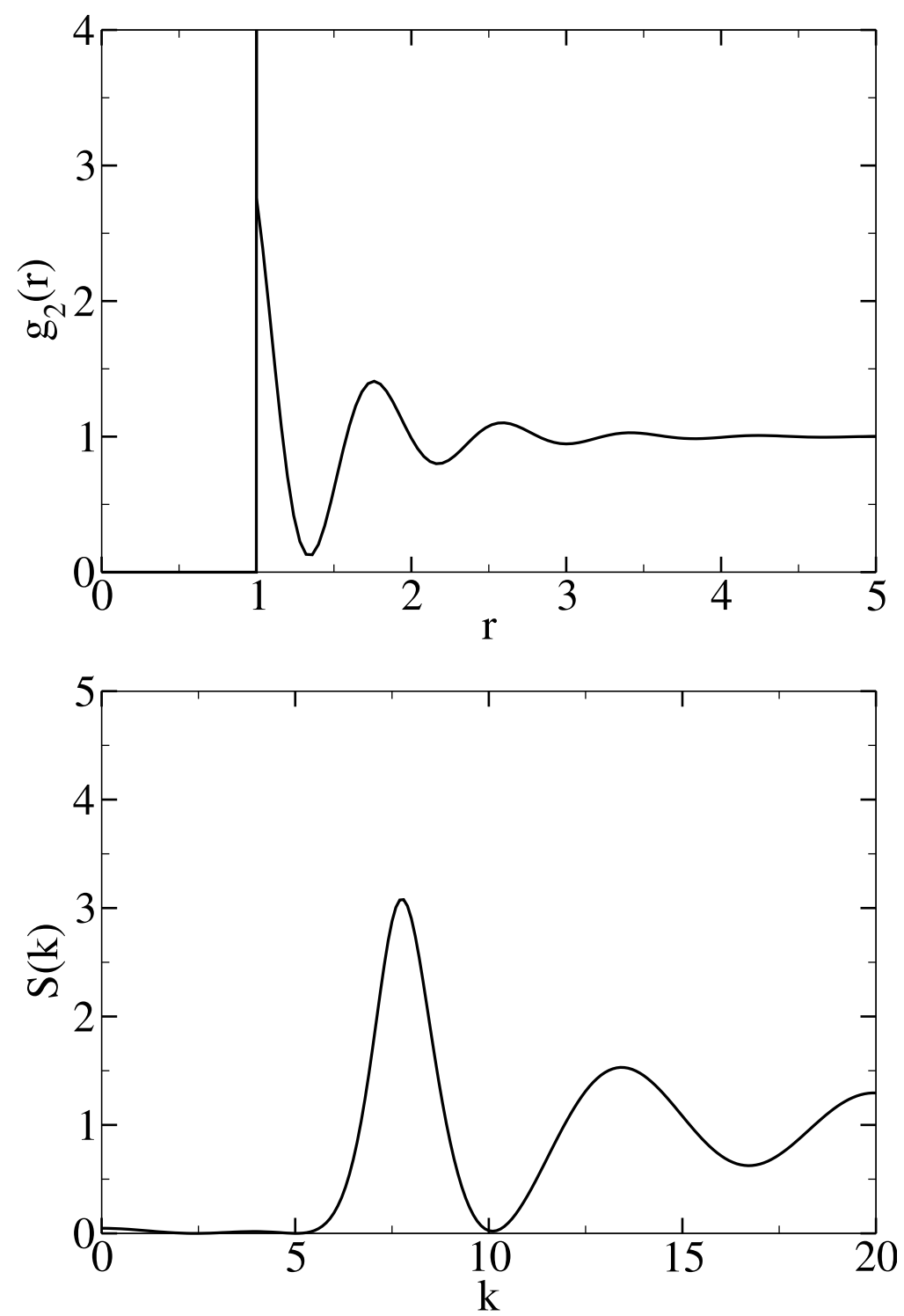

FIG. 2: Top: The pair correlation function $g_{2}(r)$ for $\phi_{*}=0.64050, A_{1}=6.1707, B_{1}=1.2090$, $C_{1}=7.6011, D_{1}=0.54981, Z=5.1593$. Bottom: The corresponding structure factor $S(k)$.

added to this form and optimized for maximum packing fraction, average kissing number increases substantially. It is of interest therefore to maximize $Z$ with packing fraction $\phi$ as a parameter. The method employed is the same as before: simulated annealing in 20,000 independent runs.

Figure 3 shows the pair correlation function for the optimal parameters $A_{1}, B_{1}, C_{1}, D_{1}$, and $\phi$, with optimized $Z=9.5401$. Most notably, the packing fraction associated with the maximum average kissing number configuration is 0.631 , nearly equal to the maximum 


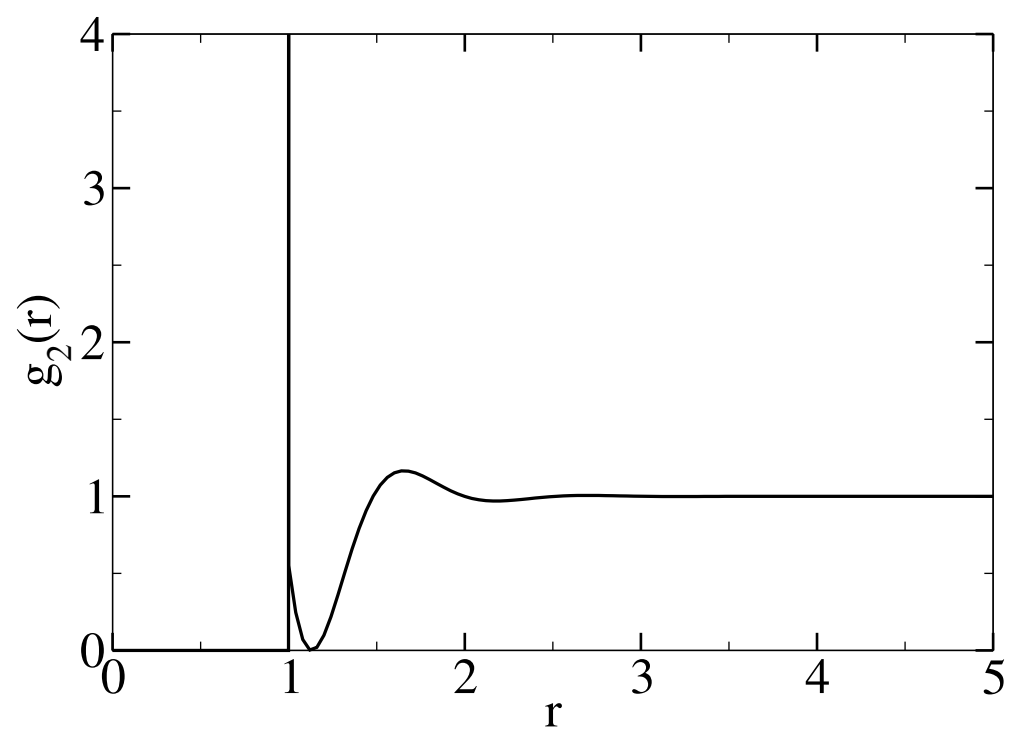

FIG. 3: The pair correlation function $g_{2}(r)$ for $Z=9.532, A_{1}=-29.02, B_{1}=2.735, C_{1}=6.0537$, $D_{1}=0.47, \phi=0.631$

packing fraction achieved $\left(\phi_{*}=0.64268\right)$ for this functional form. The fact that many more spheres are in contact (relative to the case in which the packing fraction is maximized, where $Z=5.0633$ ), could reflect the presence of large clusters in the packing or even a samplespanning cluster [33]. Also of note is that the first minimum, while still equal to zero, has moved inwards toward $r=1$, and that the value of the pair correlation function at $r=1^{+}$, $g_{2}\left(1^{+}\right)=0.5514$, is much smaller than before, where the notation $1^{+}$refers to the right hand limit of $g_{2}(r)$ as $r \rightarrow 1$.

These features must be present with an average kissing number near 12, as the integral of $g_{2}(r)$ from $r=1^{-}$to a given $R$ is related to the number of sphere centers most likely to be found in that range of distances, and for a realizable packing, this number cannot exceed 12 for $R$ near unity. Specifically, $Z(R)$, the total expected number of sphere centers to be found within a (larger) sphere of radius $R$ centered on an arbitrary sphere center within the packing, can be written

$$
Z(R)=4 \pi \rho \int_{1^{-}}^{R} x^{2} g_{2}(x) d x,
$$

where the average of $Z(R)$ as $R \rightarrow 1$ from the right $\left(1^{+}\right)$is equivalent to the average kissing number $Z$.

Generally, $Z(R)$ cannot exceed some $Z_{\max }(R)$, where $Z_{\max }(R)$ represents the maximum 
number of sphere centers that can be placed within a (larger) sphere of radius $R$ centered on an arbitrary sphere center. It is clear from geometric considerations that $Z_{\max }(R)$ is a piecewise continuous nowhere decreasing function of $R$. As the maximum number of congruent spheres that can be placed around a congruent central sphere is $12, Z_{\max }(R)$ is 12 on the interval $\{R:(1,1+\alpha)\}$ for some small deterministic parameter $\alpha>0$, where $\alpha$ is the distance from unity to the first discontinuity in $Z_{\text {max }}(R)$. Currently, $\alpha$ is not known rigorously, though its value is suspected to be about 0.045 [34]. That $Z(R)$ must be less than or equal to $Z_{\max }(R)$ for all $R$ is another necessary realizability condition for $g_{2}$ 's representing sphere packings. More will be said about this condition in the conclusions.

It is also of note that the damping parameter $B_{1}$ for the maximum average kissing number case is much larger than for the maximum packing fraction case, implying that in the maximum average kissing number case, spatial correlation decreases substantially more quickly. The configuration represented by the more heavily damped pair correlation function can be interpreted as many groups of tightly packed spheres pushed together in a random fashion, thus exhibiting a high average kissing number but little to no spatial correlation at distances greater than several sphere diameters.

\section{INCREASING PACKING FRACTION}

Here we show that the addition past contact of sinusoids decaying (to unity) as $r^{-4}$, a sinusoidally modulated form of the $r^{-4}$ decay present in pair correlation functions calculated from large-scale simulations $\left(10^{6}\right.$ spheres) of MRJ-like packings [31], to the initial fiveparameter family of $g_{2}$ 's allows for significant increase in terminal packing fraction above 0.64268. In three dimensions, the inverse $4^{\text {th }}$ power is the smallest integer power to which a pair correlation function may decay while satisfying the $r^{-3-\epsilon}$ condition for representing a disordered packing. However, aside from the clues that this mathematical attribute might provide, the structural origins of the $r^{-4}$ decay currently remain a conceptual mystery.

While the form of $r^{-4}$ decay allows increased packing fraction, other forms, including those representative of certain other features present in MRJ states, do not necessarily. In fact many other additions have been considered for this study, though from these no substantial increases in $\phi_{*}$ above the 0.64268 achieved for the five-parameter form have been obtained. This is not to say that only sinusoids decaying as $r^{-4}$ will increase the maximum 
possible attainable packing fraction, just that the selection of elements is non-trivial. In particular, one feature present in MRJ states, a fractional power-law divergence near $r=1$, resulted only in a reduced value for $\phi_{*}$. The addition of this feature is discussed in Appendix B.

The method used to optimize parameters with additional forms included involves three steps. Structure factors are calculated analytically from Eq. (14) as before, but to accommodate the increased processing time required to find the minima of $S(k)$ due to the complexity of its analytic form, the number of points $k$ at which $S(k)$ is calculated is initially reduced. The first step then is to find several "rough" maximum density configurations, just as with the initial five-parameter configurations, using a reduced number of calculations with initial parameters selected as before in 10,000 independent runs. The second step is to improve upon these approximate maxima by increasing the number density of points $k$ calculated in $S(k)$ about its minimum, in 1,000 additional runs for each rough maximum $\phi$. The results of the second step still do not yield an exact maximum and hence the third step is to finetune the maximum from the second step manually, ensuring that $S(k)$ and $g_{2}(r)$ are indeed greater than zero for all $r$ and $k$.

Sinusoids decaying as $r^{-4}$ are highly successful in increasing maximum packing fraction beyond 0.64268. Specifically, cosine functions of the form

$$
g_{I V}(r)=\frac{A}{r^{4}} \cos (B r+C) \Theta(r-1)
$$

are employed. Adding two of these elements to the initial five-parameter form allows identification of a function obeying all conditions with a maximum packing fraction of 0.6850 , $45 \%$ closer to the packing fraction of the FCC configuration than 0.640. Function (17) is the 11-parameter pair correlation functional form specified.

$$
\begin{aligned}
g_{2}(r) & =\frac{Z}{4 \pi \rho} \delta(r-1)+\left(1+\frac{A_{1}}{r} \exp \left(-B_{1} r\right) \sin \left(C_{1} r+D_{1}\right)\right) \Theta(r-1) \\
& +\left(\frac{A_{2}}{r^{4}} \cos \left(B_{2} r+C_{2}\right)+\frac{A_{3}}{r^{4}} \cos \left(B_{3} r+C_{3}\right)\right) \Theta(r-1),
\end{aligned}
$$

or

$$
g_{2}(r)=g_{I}(r)+g_{I I}(r)+g_{I I I}(r)+g_{I V a}(r)+g_{I V b}(r),
$$

where the subscripts $a$ and $b$ in the last two terms on the right refer to the fourth and fifth terms in (17). The analytical structure factors for the pair correlation functions represented by function (17) calculated using relation (5) are given in Appendix A. 


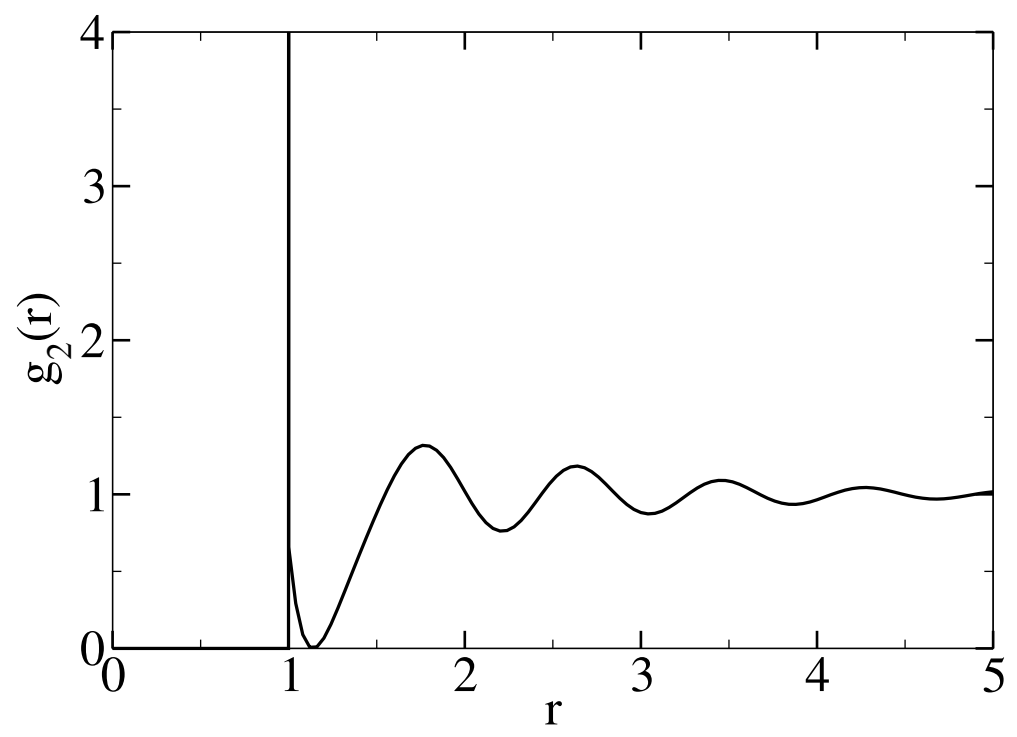

FIG. 4: The pair correlation function $g_{2}(r)$ for $\phi_{*}=0.6850, A_{1}=3.555, B_{1}=0.7189, C_{1}=7.5589$, $D_{1}=0.6247, Z=10.4281, A_{2}=0.2205, B_{2}=2.370, C_{2}=0.000, A_{3}=2.2822, B_{3}=8.7373$, $C_{3}=0.0423$

Figures (4) and (5) show graphs of the pair correlation function and structure factor with the 11 parameters of function (17) optimized for a maximum packing fraction of 0.6850 . The first minimum and height of $g_{2}(r)$ just past $r=1$ in Fig. 4 are similar to those observed in Fig. 3, the plot of the pair correlation function for a maximized average kissing number using the initial five-parameter functional form. The period $2 \pi / C_{1}$, phase $D_{1}$, and decay factor $B_{1}$ of the exponentially decaying sinusoidal function are similar to those observed in the top plots of Figs. 1 and 2, the plots of the pair correlation functions for maximized packing fraction using the initial five-parameter functional form. The minimum and height just past $r=1$ must be similar for physical meaningfulness due to high average kissing number $Z=10.4281$, while the similarities in period, phase, and decay factor indicate that a higher degree of order is maintained farther from $r=1$.

The decay factor, $B_{1}=0.7189$, for the 0.6850 maximum packing fraction function is in fact larger than the decay factor, $B_{1}=0.58091$, for the 0.64268 packing fraction function, but fast decay in the former $g_{2}$ function is avoided through the addition of the $r^{-4}$ sinusoids.

The average kissing number $Z=10.4281$ for the 0.6850 maximum packing fraction function is substantially higher than that obtained for the 0.6427 maximum packing fraction 


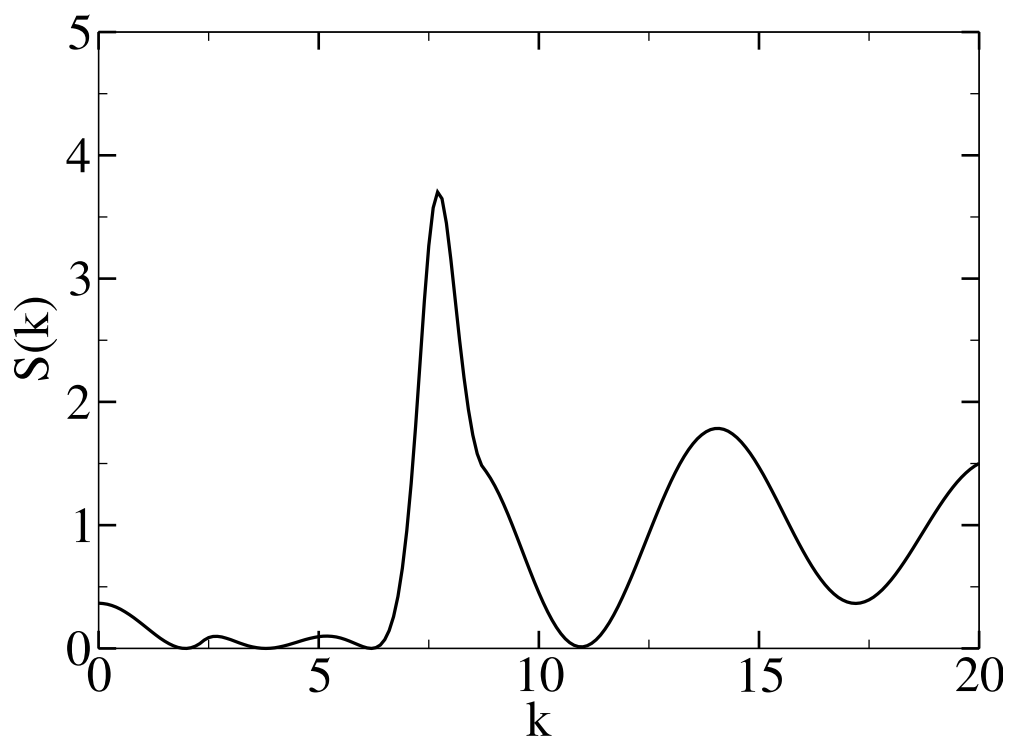

FIG. 5: The structure factor $S(k)$ for $\phi_{*}=0.6850, A_{1}=3.555, B_{1}=0.7189, C_{1}=7.5589$, $D_{1}=0.6247, Z=10.4281, A_{2}=0.2205, B_{2}=2.370, C_{2}=0.000, A_{3}=2.2822, B_{3}=8.7373$, $C_{3}=0.0423$

function. The addition of the $r^{-4}$ elements allows not only for tighter packing of spheres (and hence a higher $Z$ than previously possible), but also for slower decay than present with an exponentially decaying function alone. This implies (but does not prove) that more correlation at greater distances is necessary for higher packing fraction disordered packings: a hypothesis that will be supported further later in this paper by order metric calculations.

It is of note that reducing the packing fraction $\phi$ without proportionally reducing the kissing number $Z$ quickly violates the structure factor condition. This implies that kissing numbers as high as 10.42 cannot be maintained without proportionally high densities, which is in agreement with physical intuition: average number of spheres in contact cannot increase past a certain point without high enough packing fraction, though it is important to note that the converse of this statement is not true (if a configuration is not required to be jammed).

Further supporting this notion is Fig. 6, a graph of the pair correlation function with 10 parameters optimized to maximize $\phi$ with $Z$ set to 6.672 . With the four parameters of function (12) nearly the same as for the 0.6850 terminal packing fraction function but $Z$ reduced substantially, only a lower terminal packing fraction is possible. The amplitudes of 


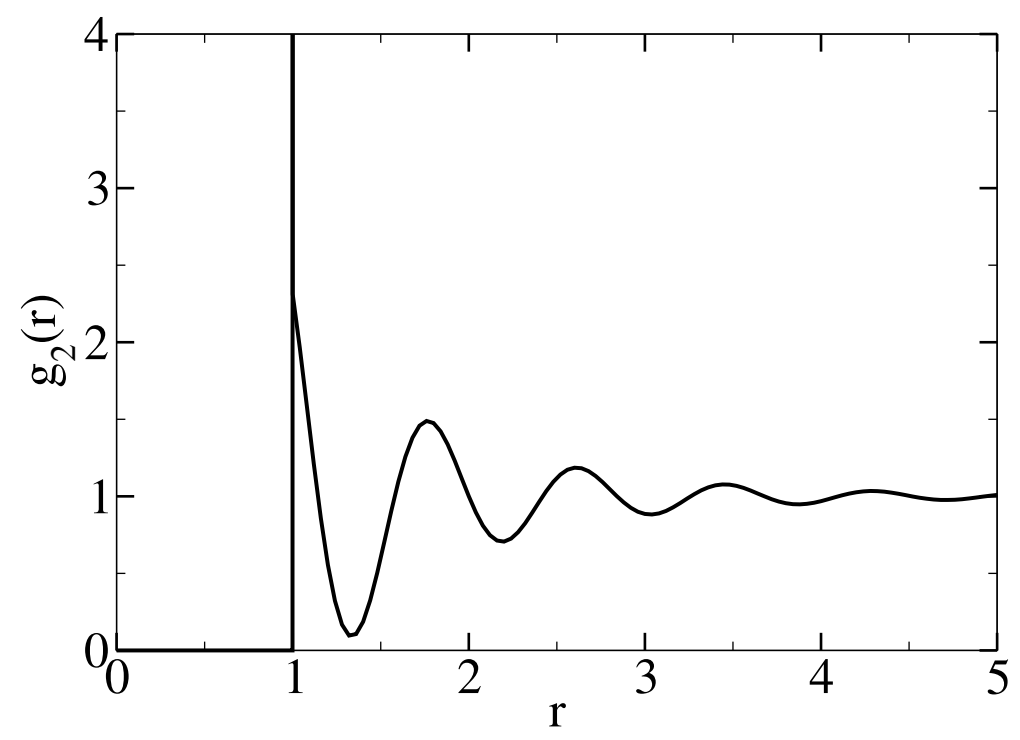

FIG. 6: The pair correlation function $g_{2}(r)$ for $\phi_{*}=0.6700, A_{1}=3.3048, B_{1}=0.71901, C_{1}=$ 7.5115, $D_{1}=0.6610, Z=6.672, A_{2}=0.0795, B_{2}=2.390, C_{2}=0.000, A_{3}=0.312, B_{3}=8.389$, $C_{3}=0.000$

the $r^{-4}$ cosine elements are also substantially reduced in Fig. 6, implying that the increase in packing fraction from 0.6700 to 0.6850 necessitates both correlation at greater distances and a substantially higher average kissing number. As will be quantified later in this paper, it follows that more order overall is required to attain the packing fraction 0.6850; nonetheless, the smooth correlation function past contact and swift decay to unity indicates that the configuration remains disordered as defined.

As before with the five-parameter functional form, it is of interest to maximize kissing number $Z$ for the 11-parameter functional form (17). We find that the average kissing number cannot increase much above 10.4281. For this functional form and with realizability conditions satisfied, we see that if one of either the packing fraction or kissing number is near its maximum value, then the other must also be near its maximum. 


\section{FROM DISORDERED TO CRYSTALLINE}

\section{A. Order metrics}

A disordered packing as defined clearly lacks the degree of order present in a periodic packing, but the degree of order within a disordered system still varies, and may be quantified. For cases of hard-sphere packings, many different methods of quantifying the order in a configuration are possible. The majority of these methods advocate the use of scalar statistical measures. Some of these assume that the most ordered system is the appropriate maximally dense packing [3, 10, 18, 19, 35], while others do not presuppose a reference crystal state [3, 18, 19, 26, 36].

For our purposes, the translational order metric introduced by Truskett et al. [18] is convenient because it is given in terms of the total correlation function:

$$
\mathcal{T} \equiv \frac{1}{\chi_{c}-\rho^{1 / 3} D} \int_{\rho^{1 / 3} D}^{\chi_{c}}\left|h\left(\chi \rho^{-1 / 3}\right)\right| d \chi
$$

where $D=1$ is the diameter of the hard spheres, $\chi=r \rho^{1 / 3}$, and $\chi_{c}$ is a selected cutoff distance [18]. The rescaled radial coordinate $\chi$ is set such that packing fractions of varying densities will be comparable, in that the total number of sphere centers over the integration range in packings of varying densities will be the same. The quantity $\chi_{c}$ must be chosen with care: in a short-ranged disordered system, if $\chi_{c}$ is chosen too large, the metric will not discriminate well between states as $h(\chi) \rightarrow 0$ with $\chi \rightarrow \infty$. If $\chi_{c}$ is chosen too small, $\mathcal{T}$ will not take contributions from correlations at greater $r$ into account. For the purpose of measuring order in all of the relatively short-range pair correlation functions described in this paper, $\chi_{c}=4$ (or for packing fraction near 0.65, about $r=3.75$ ) is chosen to ensure that

all fluctuations of the total correlation functions about zero greater than a certain minimal amplitude (about 0.1) are taken into account.

Using the translational order metric (19), we show here that the degree of order in the configurations presented is greatest for the maximum packing fraction achieved. Table I, referenced by figure number and $g_{2}(r)$ functional form, provies the average kissing number $Z$ and terminal packing fraction $\phi_{*}$ versus order $\mathcal{T}$ for the pair correlation functions previously discussed. The table conveys that large differences in order may exist for similar densities, as is the case with the pair correlation functions represented by the top plots of Figs. 1 and 2 . Additionally, a higher average kissing number does not necessitate higher order: the degree 
of order in the maximum average kissing number case of the five parameter form (Fig. 3) is substantially lower than in the the maximum packing fraction case (Fig. 1). Here, greater correlation at a distance is sacrificed to put more particles in contact. Finally, it is of note that for the maximum packing fraction cases of the five- and 11-parameter forms, represented by the top plot of Fig. 1, and Fig. 4, respectively, order is greatest. These results are by no means conclusive, but do suggest that the maximum packing fraction and maximum order representations of a given pair correlation function functional form are similar or even perhaps the same.

TABLE I: $\mathcal{T}$ order metric values

\begin{tabular}{|c|c|c|c|c|}
\hline$\phi_{*}$ & $Z$ & $\mathcal{T}$ & Fig. \# & Eq. \# \\
\hline 0.631 & 9.532 & 0.36 & 3 & 13 \\
\hline 0.64050 & 5.1593 & 0.34 & 2 & 13 \\
\hline 0.64268 & 5.0633 & 0.43 & 1 & 13 \\
\hline 0.670 & 6.672 & 0.40 & 6 & 18 \\
\hline 0.6850 & 10.4281 & 0.46 & 4 & 18 \\
\hline
\end{tabular}

\section{B. Moving toward crystalline order}

In the optimization study above only two sinusoids decaying as $r^{-4}$ were added to the initial five parameter form. We now show that an infinite (or finite) number of sinusoids decaying as $r^{-4}$, along with a Heaviside step function centered at $r=1$, can represent any bounded (nowhere infinite) piecewise-differentiable pair correlation function that decays to unity sufficiently fast. This follows directly from the existence of Fourier transforms, as will be explained shortly in greater detail, assuming that we explicitly state the bounding and decay conditions on the pair correlation function to mean that the Fourier transform of $r^{4} f(r)=r^{4}\left(g_{2}(r)-\Theta(r-1)\right)$ exists.

From a Fourier integral theorem, a radial function [37] $f(r)$ can be represented on an infinite interval not containing zero, for our purposes, $[1, \infty)$, by a continuum of sinusoids decaying as $r^{-4}$, so long as the one-dimensional Fourier transform of $r^{4} f(r)$ over the interval 
exists in the sense of generalized functions [38], as follows:

$$
f(r)= \begin{cases}\int_{0}^{\infty} \tilde{F}(k) \frac{\cos (k r)}{r^{4}} d k, & r \geq 1 \\ 0, & r<1\end{cases}
$$

with

$$
\tilde{F}(k)=\frac{1}{\pi} \int_{1}^{\infty} x^{4} f(x) \cos (k x) d x .
$$

Under these conditions, a smooth disordered hard-sphere $g_{2}$ that decays to unity can be written simply as

$$
g_{2}(r)=f(r)+\Theta(r-1) .
$$

For example, to represent $g_{I V}(r)$ (Eq. (16)) in this form with $C=0$, using the parameters from Eq. 16 , we set $\tilde{F}(k)=A \delta(k-B)$.

Discretizing the $k$ in expression (21) with increment $\Delta k$ between two successive discrete values, $f(r)$ may be approximated with $\tilde{F}(k)$ a series of real constants. In the limit as $\Delta k \rightarrow 0$ or as $k$ becomes continuous, expression (20) holds and any $g_{2}(r)$ meeting the aforementioned conditions may be represented by Eq. (22). Thus, to demonstrate that disordered packings as defined can attain densities up to $\pi / \sqrt{18}$, it only remains to show that there is a realizable packing with packing fraction near $\pi / \sqrt{18}$ that can be represented by a pair correlation function in the form of Eq. 22 for which the Fourier transform of $r^{4} f(r)$ exists, which is the focus of the remainder of this section.

The pair correlation function of an infinite close-packed FCC crystal consisting of congruent spheres of diameter $D=1$ can be written as

$$
g_{2}^{f c c}(r)=\frac{1}{4 \pi \rho} \sum_{i=1}^{\infty} \frac{C_{i}^{f c c}}{q_{i}^{2}} \delta\left(r-q_{i}\right),
$$

where the $q_{i}$ are the distances from the origin to each successive shell in an FCC packing (which in these scaled coordinates are $q_{i}=\sqrt{i}$ ), the sum runs only over those $i$ for which shells are present (for example, $i=14,30,46$, etc. are skipped), the $C_{i}^{f c c}$ are the coefficients of the (even-exponent) terms in the FCC theta series [1] (which represent the number of spheres present on a shell), and $\delta$ represents a Dirac delta function. Any same-density stacking variant of an FCC configuration, i.e., any of the Barlow packings [39, 40], may be represented by a similar series of delta functions. For a spherical crystal of finite size, the $q_{i}$ remain the same but terminate for $i>2 I$ where $\sqrt{I}$ is the distance from the central sphere 
center to the center of the outermost sphere of the crystal, and the $C_{i}$ must be reduced to reflect a lower average number of spheres present at given distances for all but the central sphere.

An FCC configuration is not, however, disordered. To create a disordered packing from a series of finite-size FCC crystals, three further steps must be taken. First, the delta functions are replaced with smooth, sharply peaked Gaussian-like curves that decay to zero at distance $|w / 2|$ from each $q_{i}$. We define the integral under each of these curves from $q_{i}-w / 2$ to $q_{i}+w / 2$ to be equal to the reduced $C_{i}$ of a FCC crystal of specified finite size. Physically, this represents packing spheres of diameter 1 in a FCC arrangement, shrinking the radius of these spheres to $r_{a}=(1 / 2)-(1 / 4) w$, and then moving each sphere a distance of no more than $(1 / 4) w$ from its initial position such that the pair correlation function of the packing near each $q_{i}$ has the properties just described. It is of note that the exact form of the pair correlation function is not of consequence as long as the proscribed properties are maintained.

Second, the $C_{i}$ are again altered such that the finite-size "shrunk" FCC configuration of spheres becomes cubical in shape, with a cube of side length $I^{\text {cube }}$ circumscribing the arrangement. Finally, an infinite number of these cubes, with principal axes of the FCCpacked spheres within each cube arranged at uncorrelated angles, are packed together tightly such that each pair of parallel faces of every cube are parallel to a pair of faces on every other cube, but with each face touching four other faces (i.e., four other cubes). Cubes may be tightly packed in this fashion such that they fill space, and such that the resulting pair correlation function of the spheres comprising these cubes is disordered due to the packing of the cubes and the random arrangement of the principal axes of the FCC-packed spheres within each cube.

This pair correlation function would be in practice difficult to write explicitly due to boundary effects, but this is inconsequential to the description, as all that is necessary is to illustrate that such packings exist. Additionally, it is noteworthy that initially packing the spheres in each cube in arrangements equivalent to any of the Barlow packings would yield an equivalent result.

Boundary effects become negligible as the number of cubes approaches infinity, and for increasing $I^{c u b e}$ and decreasing $w$, any packing fraction $\pi / 6<\phi<\pi / \sqrt{18}$, with $\pi / 6=\phi^{s c}$ the packing fraction of a simple cubic arrangement, can be obtained. The minimal packing 
fraction $\phi^{s c}$ is obtained when only one sphere is present in each cube. The distance $w$ can be made small enough for any finite-sized system of cubes such that the system exhibits physical stability when force is applied, though the pair correlation function of such a system would change (perhaps to include delta functions) as soon as stress and strain were present.

In this way, as long as $w>0$ and $I^{\text {cube }}$ is finite, a disordered packing may exhibit any packing fraction up to $\pi / \sqrt{18}$, if one accepts that the pair correlation function of the system described above, represented in the form of Eq. (22), decays to unity at least as fast as $r^{-3-\epsilon}$ and in a form such that the Fourier transform of $r^{4} f(r)$ exists. Due to this result and the others demonstrated in this paper, we state that the sequential addition and optimization of more than two sinusoidal terms of the form of expression 16 will allow packing fractions greater than 0.6850 , and as the number of terms grows, the optimal packing fraction for a realizable $g_{2}$ is conjectured to approach $\pi / \sqrt{18}$.

\section{CONCLUSIONS AND DISCUSSION}

Using the $g_{2}$-invariant method with $g_{2}$ 's satisfying the three necessary, but generally not sufficient, conditions for realizability, we demonstrated without implicit reliance upon any packing methodology that packing fractions well above 0.64 are obtainable for pair correlation functions incorporating the salient features of disordered packings. A packing fraction of 0.6850 was obtained employing a test family of $g_{2}$ 's mimicking features observed in MRJ packings, including most notably core exclusion, contact pairs, and a sinusoidal decay to unity as $r^{-4}$. Consistent with a previous study [10], we found that to achieve higher packing fractions, the degree of order must increase.

Additionally we showed, employing a qualitative example and a revised version of a Fourier integral theorem, the surprising result that a disordered packing as defined may reach packing fractions approaching $\pi / \sqrt{18}$, the maximum possible for a three-dimensional hard-sphere packing. These results support the hypothesis that continued addition and subsequent optimization of sinusoids decaying as $r^{-4}$ (of the form of Eq. (16)) will find realizable $g_{2}$ with higher terminal packing fractions up to (but not reaching) $\pi / \sqrt{18}$.

The conclusion that the addition of sinusoids decaying as $r^{-4}$ allows higher terminal packing fractions (where the addition of other features does not necessarily) is very relevant to the study of high-density physical and jammed disordered systems, since it demonstrates 
that this feature contributes significantly in allowing the systems to reach aforementioned higher densities. It is noteworthy that the $r^{-4}$ decay to unity present in the $g_{2}$ 's of MRJ packings also characterizes $g_{2}$ 's of high-density Bose systems [41], ground states of fermionic systems [42], and models of the density distribution of the early Universe [43, 44, 45, 46], though these latter systems are not sphere packings.

In future work, we will seek to extend these results to configurations of multi-component spheres and non-spherical objects. Additionally, we will investigate the structural origins of the presence of the $r^{-4}$ decay in MRJ states, and attempt to determine if in higher dimensions additions of terms consisting of sinusoids decaying as the smallest (inverse) integer power for which a packing remains disordered, $r^{-d-1}$, to a $g_{2}(r)$ representing the salient features of a $d$-dimensional hard sphere system, will allow for packing fractions up to the known maximum in that dimension.

Moreover, we will examine the form of $Z_{\max }(R)$, the maximum number of sphere centers that can be placed within a (larger) sphere of radius $R$ centered on an arbitrary sphere center. A natural question to ask is whether the realizability condition $Z(R) \leq Z_{\max }(R)$ further constrains $g_{2}(r)$ beyond the three conditions (2), (3), and (7). The answer is in the affirmative. We have already noted that the Cohn-Elkies linear programming upper bound formulation [22] is the dual of the Torquato-Stillinger lower bound procedure, i.e., the $g_{2^{-}}$ invariant process [21]. Cohn and Kumar [47] recently proved that there is no duality gap between the primal and dual LP programs, which means both the upper and lower bounds coincide when the best test functions are employed. Cohn and Elkies were able to find the test functions that yield the best upper bound on the maximal packing fraction in three dimensions: a packing fraction of about 0.778, which is well above the true maximal value. This means that there exists a test pair correlation function for the lower bound formulation that will deliver the same maximal packing fraction of 0.778 , which clearly is not realizable. It was shown elsewhere that applying the additional condition that $Z_{\text {max }}$ must be equal to 12 up to some small positive $\alpha$ beyond contact together with the best test function in the upper bound brought down the maximal packing fraction appreciably from 0.778 [48]. This means that adding the $Z_{\max }$ condition to the corresponding best test pair correlation function will also improve the packing fraction estimate. Therefore, the $Z_{\max }$ condition introduces additional information beyond that contained in the two standard nonnegativity conditions. The precise form of this additional condition has yet to be fully elucidated and 
will be explored in future work.

\section{Acknowledgments}

The authors are grateful to Henry Cohn for a critical reading of our manuscript. S.T. thanks the Institute for Advanced Study for its hospitality during his stay there. This work was supported by the Division of Mathematical Sciences at the National Science Foundation under Award Number DMS-0804431 and by the MRSEC Program of the National Science Foundation under Award Number DMR-0820341.

\section{APPENDIX A: ANALYTICAL STRUCTURE FACTOR COMPONENTS}

The analytical structure factor components, as calculated from relation (5), for $g_{I}, g_{I I}$, $g_{I I I}, g_{I V}$ and $g_{V}$, with $r=1$ the diameter of the spheres.

$$
\begin{gathered}
\mathbf{G}_{\mathbf{I}}(k)=\frac{4 \pi}{k^{3}}(k \cos k-\sin k) \\
\mathbf{G}_{\mathbf{I I}}(k)=\frac{Z}{\rho k} \sin k \\
\mathbf{G}_{\mathbf{I I I}}(k)=\frac{2 \pi A e^{-B}}{k} \\
\left(\frac{B \cos (k-C-D)-(k-C) \sin (k-C-D)}{B^{2}+(k-C)^{2}}-\right. \\
\left.\frac{B \cos (k+C+D)-(k+C) \sin (k+C+D)}{B^{2}+(k+C)^{2}}\right)
\end{gathered}
$$

$$
\begin{aligned}
& \mathbf{G}_{\mathbf{I V}}(k)=\frac{A \pi}{2 k}\left(\operatorname { c o s } C \left[\pi(B-k)|B-k|-\pi(B+k)^{2}\right.\right. \\
& \left.+2(B+k)^{2} \operatorname{Si}(B+k)-2(B-k)^{2} \operatorname{Si}(B-k)\right] \\
& +\sin C\left[2(B+k)^{2} \operatorname{Ci}(B+k)+(B-k)^{2}(2 \log (B-k)\right. \\
& \left.\left.-\log (B-k)^{2}-2 \operatorname{Ci}(B-k)\right)\right]+4 k \cos k \cos (B+C) \\
& +4 \sin k[\cos (B+C)-B \sin (B+C)])
\end{aligned}
$$




$$
\begin{aligned}
& \mathbf{G}_{\mathbf{V}}(k)=\frac{8 A \sqrt{B} \pi}{15}\left(k B \operatorname { c o s } k \left[5_{1} \mathrm{~F}_{2}\left(\frac{3}{4} ; \frac{3}{2}, \frac{7}{4} ;-\frac{1}{4} k^{2} B^{2}\right)\right.\right. \\
& \left.+3 B_{1} \mathrm{~F}_{2}\left(\frac{5}{4} ; \frac{3}{2}, \frac{9}{4} ;-\frac{1}{4} k^{2} B^{2}\right)\right]+\left[3{ }_{1} \mathrm{~F}_{2}\left(\frac{1}{4} ; \frac{1}{2}, \frac{5}{4} ;-\frac{1}{4} k^{2} B^{2}\right)\right. \\
& \left.\left.+B_{1} \mathrm{~F}_{2}\left(\frac{3}{4} ; \frac{1}{2}, \frac{7}{4} ;-\frac{1}{4} k^{2} B^{2}\right)\right] 5 \sin k\right)
\end{aligned}
$$

In the expression for $G_{I V}(k), \operatorname{Si}(k)$ represents the standard sine integral, $\operatorname{Si}(k)=$

$\int_{0}^{k} d x \sin x / x$, and $\mathrm{Ci}(k)$ represents the standard cosine integral, $\mathrm{Ci}(k)=-\int_{k}^{\infty} d x \cos x / x$. In the expression for $G_{V}(k),{ }_{p} \mathrm{~F}_{q}\left(\left\{a_{i}\right\},\left\{b_{j}\right\}, k\right)$ represents the standard hypergeometric ${ }_{p} \mathrm{~F}_{q}$ function,

$$
{ }_{p} \mathrm{~F}_{q}\left(\left\{a_{i}\right\},\left\{b_{j}\right\}, k\right)=\sum_{n=0}^{\infty} \frac{\left(a_{1}\right)_{n} \cdots\left(a_{p}\right)_{n} k^{n}}{\left(b_{1}\right)_{n} \cdots\left(b_{q}\right)_{n} n !},
$$

with $\left(a_{i}\right)=\gamma(a+i) / \gamma(a)$ and $\gamma(x)$ the standard gamma function.

\section{APPENDIX B: POWER-LAW DIVERGENCE IN NEAR CONTACT DISTRI-}

\section{BUTION}

Evidence indicates that the addition of one of the salient features observed in MRJ-like states for three-dimensional hard-sphere packings, a fractional power-law divergence near $r=1$ due to near contacts [49, 50], does not increase packing fraction past that obtained from the five-parameter form. For example, the addition of the form

$$
g_{V}(r)=\frac{A_{4}}{(r-1)^{1 / 2}} \Theta(r-1) \Theta\left(B_{4}-r\right),
$$

with $B_{4}=1.15$ a cutoff parameter to the square-root power decay, to the $g_{I}(r), g_{I I}(r)$, $g_{I I I}(r)$ discussed earlier led to no additional increase in packing fraction under maximum density parameter optimization in 10,000 independent runs. Figure 7 shows a graph of the maximum packing fraction obtained versus the value of coefficient $A_{4}$, where values plotted represent a random sample of 100 from the 10,000 runs conducted. One can see clearly that as $A_{4}$ increases in value, indicative of the increased prominence of the inverse square-root divergence near $r=1$, maximum packing fraction obtained decreases steadily.

These results imply that the inverse half power decay near $r=1$ is a feature intrinsic to MRJ configurations and that this feature must be diminished to increase packing fraction. Physically this is intuitive, as the presence of a half power decay near $r=1$ indicates that 


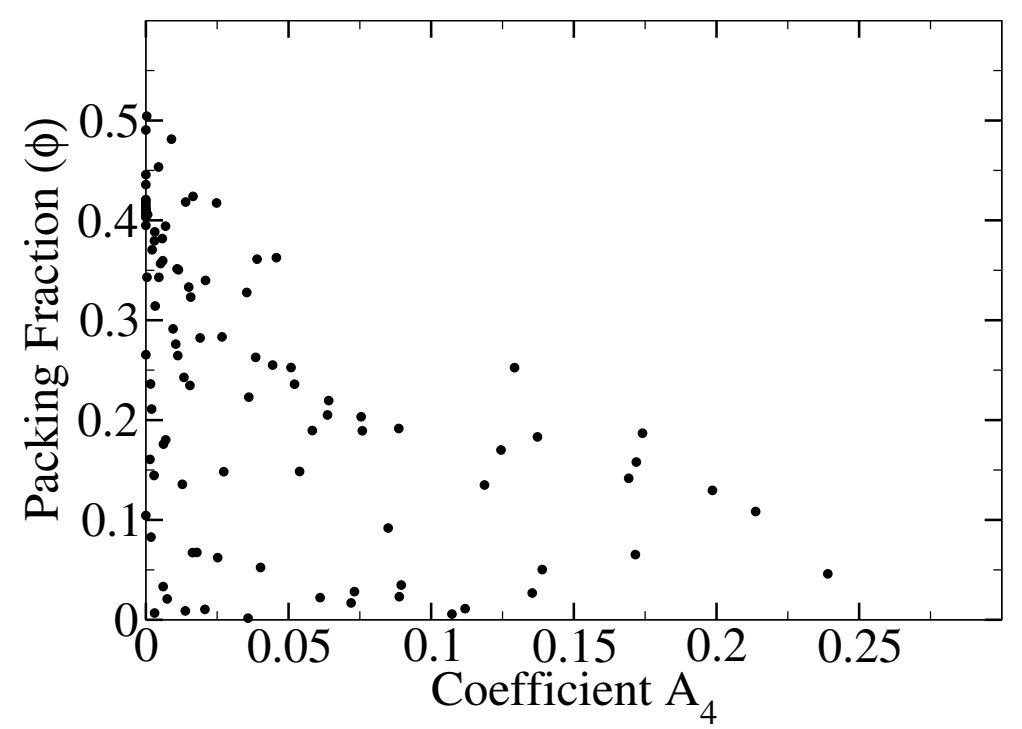

FIG. 7: Maximum obtained packing fraction $\phi$ versus value of parameter $A_{4}$, represented by a random selection of 100 from 10,000 maximum density parameter optimization runs where a term $g_{V}(r)$, given by relation (B1), was added to the initial five-parameter pair correlation function functional form 13

there are many spheres smoothly distributed just outside of contact, i.e., that locally on average there is room to compress the system further.

[1] J. H. Conway and N. J. A. Sloane, Sphere Packings, Lattices and Groups (Springer, 1999).

[2] T. Hales, Ann. Math. 162, 1065 (2005).

[3] S. Torquato, Random Heterogeneous Materials - Microstructure and Macroscopic Properties (Springer-Verlag, New York,, 2002).

[4] S. Torquato, Int. J. Solids Structures 35, 2385 (1998).

[5] S. F. Edwards, Granular Matter (Ed. A. Mehta, Springer-Verlag, New York, 1994).

[6] P. M. Chaikin and T. C. Lubensky, Principles of Condensed Matter Physics (Cambridge University Press, New York, 2000).

[7] T. I. Zohdi, Mech. Mater. 38, 969 (2006).

[8] J. Bernal, Nature 183, 141 (1959).

[9] G. D. Scott, Nature 188, 908 (1960). 
[10] S. Torquato, T. M. Truskett, and P. G. Debenedetti, Phys. Rev. Lett. 84, 2064 (2000).

[11] S. Torquato and F. H. Stillinger (unpublished).

[12] G. D. Scott and D. M. Kilgour, Br. J. Appl. Phys. Series 2 2, 863 (1969).

[13] J. L. Finney, Proc. Roy. Soc. Lond. A 319, 479 (1970).

[14] J. D. Bernal and J. L. Finney, Proc. Roy. Soc. Lond. A 319, 495 (1970).

[15] O. Pouliquen, M. Nicolas, and P. D. Weidman, Phys. Rev. Lett. 79, 3640 (1997).

[16] B. D. Lubachevsky and F. H. Stillinger, J. Stat. Phys. 60, 561 (1990).

[17] B. D. Lubachevsky, F. H. Stillinger, and E. N. Pinson, J. Stat. Phys. 64, 501 (1991).

[18] T. M. Truskett, S. Torquato, and P. G. Debenedetti, Phys. Rev. E 62, 993 (2000).

[19] A. R. Kansal, S. Torquato, and F. H. Stillinger, Phys. Rev. E 66, 041109 (2002).

[20] S. Torquato and F. H. Stillinger, J. Phys. Chem. B 106, 8354 (2002).

[21] S. Torquato and F. H. Stillinger, Experimental Mathematics 15, 307 (2006).

[22] H. Cohn and N. Elkies, Ann. of Math. 157, 689 (2003).

[23] F. Berrilli, D. Del Moror, G. Consolini, E. Pietropaolo, T. L. Duvall, Jr., and A. G. Kosovichev, Solar Phys. 221, 33 (2004).

[24] T. Kuna, J. L. Lebowitz, and E. R. Speer, J. Stat. Phys. 129, 417 (2007).

[25] M. Yamada, Prog. Theor. Phys. 25, 579 (1961).

[26] S. Torquato and F. H. Stillinger, Phys. Rev. E 68, 041113 (2003).

[27] A. Scardicchio, F. H. Stillinger, and S. Torquato, J. Math. Phys. 49, 043301 (2008).

[28] J. Crawford, S. Torquato, and F. H. Stillinger, J. Chem. Phys. 119, 7065 (2003).

[29] O. U. Uche, F. H. Stillinger, and S. Torquato, Physica A 360, 21 (2006).

[30] The requirement for smoothness past contact can be expressed mathematically by stating that $g_{2}(r)$ is a $C^{n}$ function with $n \geq 1$, or that at least its first derivative exists for $r>1$.

[31] A. Donev, F. H. Stillinger, and S. Torquato, Phys. Rev. Lett. 95, 090604 (2005).

[32] J. Leech, The Mathematical Gazette 40, 22 (1956).

[33] A cluster is a group of contacting particles in which there is a connected path lying inside all of the particles of the group that never enters the space exterior to the particles.

[34] K. Schutte, and B. L. van der Waerden, Math. Annalen. 123, 96 (1951).

[35] P. J. Steinhardt, D. R. Nelson, and M. Ronchetti, Phys. Rev. B 28, 784 (1983).

[36] L. T. To, D. J. Daley, and Z. H. Stachurski, Solid State Science 8, 868 (2006).

[37] A radial function $f(r)$ on $\mathbb{R}$ in dimension $d$ depends only on the radial distance $r=|\mathbf{r}|$, where 
$\mathbf{r}$ is the vector position in $\mathbb{R}^{d}$.

[38] R. V. Churchill, Fourier Series and Boundary Value Problems (McGraw-Hill, 1956).

[39] W. Barlow, Nature 29, 186 (1883).

[40] The Barlow packings include all permutations of the stackings of three specific planes of spheres, $A, B, C$, where none of the three stackings is adjacent to its like (no $A A, B B, C C$ ), of which FCC and $\mathrm{HCP}$ are special cases, stacked $A B C A B C \ldots$ and $A B A B \ldots$, respectively.

[41] L. Reatto and G. V. Chester, Phys. Rev. 155, 88 (1967).

[42] S. Torquato, A. Scardicchio and C. E. Zachary, J. Stat. Mech.: Theory and Experiment, P11019, (2008).

[43] E. R. Harrison, Phys. Rev. D 1, 2726 (1970).

[44] Y. B. Zeldovich, Mon. Not. R. Astron. Soc. 160, 1 (1972).

[45] P. J. E. Peebles and J. T. Yu, Astrophysical J. 162, 815 (1970).

[46] A. Gabrielli, M. Joyce, and F. S. Labini, Phys. Rev. D 65, 083523 (2002).

[47] H. Cohn and A. Kumar (unpublished).

[48] H. Cohn, A. Kumar, and S. Torquato (unpublished). If $\alpha$ is assumed to be 1.05, then the packing fraction upper bound improves to about 0.771. If $\alpha=1.1$ instead, then the bound improves to 0.764 .

[49] L. E. Silbert, D. Ertas, G. S. Grest, T. C. Halsey, and D. Levine, Phys. Rev. E 65, 031304 (2002).

[50] A. Donev, S. Torquato, and F. H. Stillinger, Phys. Rev. E 71, 011105 (2005). 